Voix et Images

voixetimages

\title{
La fiction du réel / le réel de la fiction
}

\section{André Brochu}

Volume 12, numéro 2 (35), hiver 1987

Jacques Brault

URI : https://id.erudit.org/iderudit/200642ar

DOI : https://doi.org/10.7202/200642ar

Aller au sommaire du numéro

\section{Éditeur(s)}

Université du Québec à Montréal

\section{ISSN}

0318-9201 (imprimé)

1705-933X (numérique)

Découvrir la revue

\section{Citer cet article}

Brochu, A. (1987). La fiction du réel / le réel de la fiction. Voix et Images, 12(2), 322-330. https://doi.org/10.7202/200642ar d'utilisation que vous pouvez consulter en ligne.

https://apropos.erudit.org/fr/usagers/politique-dutilisation/ 


\title{
Poésie
}

\section{La fiction du réel / le réel de la fiction}

\author{
par André Brochu, Université de Montréal
}

La conscience n'a pas un accès direct au réel. Le réel est un construit, une fiction. Percevoir, ce n'est pas enregistrer passivement l'objet mais se le donner, par un acte qui est d'abord une invention. Et la poésie. grâce à l'usage qu'elle fait du langage, manifeste mieux que toute autre forme d'expression verbale la relation originaire par laquelle se créent simultanément l'homme et le monde. Le but du poète n'est pas d'abord d'établir la communication. en faisant appel à un langage homogène, "commun», mais d'écrire la "fiction du réel», selón la formule qui donne son titre à la singulière rétrospective de Fernande Saint-Martin'.

Ces propos résument partiellement l'introduction, d'une écriture tout universitaire, où l'auteur prend parti pour René Thom contre la linguistique chomskienne et toute cette tradition de pensée qui fait du langage une copie conforme des choses. Suivent les poèmes, qui en effet n'ont pas pour objectif premier la communication - sans doute est-ce la raison de leur tenue au secret: qui aurait pu prédire le surgissement d'une telle ceuvre, patiemment et régulièrement édifiée, au vu des quelques pages publićes dans Liberté (été 1965) et les Herbes rouges (printemps-été 1969)? Fernande Saint-Martin ne cherche pas à transmettre une émotion, ou des idées, mais plutôt à explorer les possibilités de ce que j’appellerais une machine rythmique et sémantique. Il s'agit en particulier de faire jouer ensemble un certain nombre d'isotopies, notamment celle du corps (un des premiers poèmes. de 1954, comprend les mots: peau, coudes, yeux, gorge, os, dents, langues; un des derniers, de 1975. réunira: ceil, paume, enflure, joue, chair, cils, ventru, séant), celle de la nature (les mêmes poèmes comprennent, respectivement: palme, gouttes. nuits, eau, farine, perles, monts, lune, marée, pommes, fleurs, diamant ; et : miel, vallées, simiesques, légumineuses, branche. soie), et l'isotopie de l'objet (1954: mur, lit, tapissant, caisses, bloc, caveau, cône; et 1975: cible, balle, voilées, clouer, fil). Le jeu consiste à combiner, de toutes les façons possibles, les significations relevant de ces isotopies, ce qui donne, par exemple:

La peau palme entre les coudes

mur de lit où les jeux achèvent

mourir la distance

gouttes riantes

tapissant la gorge dépliée

os de leau tournoyante farine (...)

Les effets de sens de cette combinatoire sont-ils de nature à faire partager au lecteur l'expérience de soi/du langage/du monde qui définit la poésie? Hélas, on peut en douter, surtout quand on constate que, au cours de vingtdeux ans, les fruits du travail se ressemblent à s'y méprendre, les seules transformations repérables étant ces variantes d'un même texte que l'auteur se plaît à fabriquer ( Variables de $A$ à $N$ ). Entre le poème et sa (ou ses) 
variante(s), qui lui est (lui sont) contemporaine(s), il.y a plus de contrastes qu'entre le poème de 1953 et le poème de 1975 - c'est dire que la seule différence possible est de nature intellectuelle, elle n'est pas relative à l'expérience humaine et poétique, qui ne semble subir aucune évolution.

Autre sujet de déception: j'ai rarement trouvé autant de fautes dans un seul livre (par exemple, celà, répété plusieurs fois, ou encore ç devant un é, quand ce n'est aggrégats, aristotélitienne, de mauvais accords du verbe avec le sujet, etc.). Je retiens une blague méchante sur les académies qui ne sont plus ce qu'elles étaient - bel exemple de prétérition!

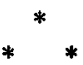

François Charron revient sous les feux de l'actualité littéraire avec un recueil relativement considérable (compte tenu d'une mise en page très aérée), le Fait de vivre ou d'avoir vécu ${ }^{2}$. Le titre suggère une poésie-témoignage. dans la veine autobiographique des derniers ouvrages du puète. Mais il faut préciser aussitôt que le je est défait (déconstruit. comme disent les cégépiens) dès que posé; rien n'est plus loin de la confidence que ces écrits en faux vers libres, tous à peu près de même dimension, regroupés en onze sections. Je parle de faux vers libres car on sent fort bien que la découpe de l'énoncé est purement contingente, comme celle du texte en prose, mais qu'il y manque ce que les typographes appellent la justification. De cette façon, François Charron joue formellement sur les deux tableaux de la poésie et de la prose, de même qu'il brouille leur frontière au plan même de l'écriture.

Le titre annonce un contenu en deux volets - vivre et avoir vécu. Deux volets point trop distincts puisque c'est le ou qui les coordonne. En fait, il s'agira d'un glissement graduel du sentiment de la vie, qui triomphe dans le premier tiers du recueil, à celui d'avoir vécu, d'être désormais face au désastre et à la mort. On observe un cheminement passablement précis qui sous-tend l'ensemble du livre. D'abord il y a le moi et sa relation à lui-même, au monde:

De toutes parts en moi la beauté contient l'être, sa libre violence, l'être qui respire et se fane.

La positivité contient en elle-même le principe de sa fin (l'être se fane) mais elle s'affirme d'abord comme plénitude et rayonnement.

Dans la deuxième section, le moi cède la place à "un personnage», qui incarne d'abord les valeurs de vie fascinante, puis soudain la vieillesse. On passe du vivre à l'avoir-vécu, et ce mouvement reproduit celui de l'ensemble car au "personnage" succèdent, dans les sections suivantes, la silhouette humaine, encore individuelle mais abstraite, puis la personne humaine, abstraite et générale, la disparition (qui est l'antonyme de l'apparition: une forme frappée de néant), et autres identités aussi vagues que n'importe qui, quelqu'un, l'écoutant, une personne, chacun, on, l'humanité, un individu, vous. Et plus la présence humaine se fait impersonnelle, plus le réel lui-même 
se fait abstrait, devenant rectangle, carré, cube. Cependant sévit, grandissante, la mort, contenue tout de même (déconstruite?...) par le pouvoir d'aimer, ou encore de parler:$$
\text { ... Malgré }
$$
cela, malgré le calcaire, malgré les
éclats de silex, nous proférons des
vérités particulières qui durent
aussi longtemps que nous vivons.

La parole est fréquemment rythmée par de brèves énumérations, le plus souvent de trois éléments: Là où il y a blocs, fonctions, / empilements de sacs: va, donne, reprends (...) Adresse-toi à l'averse, à la / fuite, aux lames... Comme toujours, la poésie de Charron donne l'impression à la fois du naturel et du fabriqué, d'une profondeur qui s'applique à se fuir comme profondeur, à s’aplatir en a-signifiances. Mais cela reste fascinant.

Denise Desautels, qui avait publié il y a quelque temps un livre admirable, l'Écran, dont la prose savante et sensible s'accordait avec les frémissements de la passion et de l'angoisse, nous revient avec un ouvrage beaucoup plus sec: la Répétition ${ }^{3}$. Les références à la tragédie et à la mythologie grecque qui constituaient la scène connotative du texte précédent passent ici à l'avant-plan et. du coup, se vident de leur substance; le théâtre fournit le motif de la répétition, qui s'applique simultanément à diverses formes de représentation. en particulier celle de "la professeure», campée dans sa triviale scénographie (Sous les regards. Clap. Clap. Clap. Clap.). Pour ce qui est de la mythologie: Dans ses lettres, il l'appelait amoureusement Antigone - entre parenthèses. C'est bien la connotation qui est mise entre parenthèses, l'espace symbolique (dénotatif) devient le lieu de croisement de toutes les isotopies, privées de leur prolongement suggestif. Plus rien n'existe que dans le temps et le lieu de son énonciation:

Je suis au cour du texte et du doute. Au cinéma. Au théâtre. Au musée. Au bout du fil qui m'interpelle en accéléré. Entre les images et les mots. Éparse et diverse. Je pense à vous si fort, vous savez. La table de travail. le papier quadrillé, la plume. L'enfant. La mère. Le monde. Éperdue.

N'y a-t-il pas là un laconisme appliqué, à la Duras, qui dessert le projet poétique parce que beaucoup plus approprié au récit, ou encore au monologue de théâtre? Sans doute le style performatif fait-il l'intérêt, par exemple, de l'écriture d'Anne-Marie Alonzo, dans Bleus de mine, mais il ne supprime pas la suggestion. Ici, on se trouve devant un effort louable de dépouillement, au plan du discours immédiat, mais l'intention proprement poétique me semble assez peu personnelle et mal réalisée. La Répétition, au titre prédestiné, donne une impression de déjà lu, de post-formalisme fatigué. Clap. 
Clap. Clap. Clap. Tant mieux si j’ai tort, car j'ai beaucoup d'admiration pour le travail habituel de Denise Desautels; et je ne doute pas, quoi qu'il en soit, de la réussite de ses prochains livres.

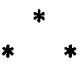

Christiane Frenette, dans la trentaine, née à Québec, remporte le prix Octave-Crémazie 1986 pour un premier recueil, Indigo nuit ${ }^{4}$. Beau livre, soigneusement écrit. La facture des poèmes est homogène, le langage manifeste de la maturité et de la maîtrise. Aucune trace de formalisme, et pourtant on ne régresse pas vers l'humanisme ni la thématique du pays. Les poèmes, de tonalité sombre, disent la difficulté de la relation à soi et aux autres, à l'autre surtout, homme ou femme (il semble y avoir un déplacement du masculin vers le féminin au cours du livre).

Ce qui frappe surtout, c'est la complexité d'un monde où les contraires sont constamment en présence l'un de l'autre. Par exemple, dès le premier poème, la violence des éléments est à la fois déniée et rendue plus crédible par la contre-hyperbole initiale:

ce n'était pas la fin du monde des hivers en furie

nous tournaient autour nous avions

l'impression de nous fracasser contre la vitre

quelquefois je t'entendais chanter

Les pulsions sémantiques positives et négatives se succèdent abruptement, et même dans les poèmes plus uniformément nocturnes, où perce mal l'enchantement de l'indigo, le désespoir reste musclé, vécu par tout le corps - loin de ce désespoir de tête, si absolu, si parfait, qui caractérise souvent la poésie des universitaires...

Ces qualités ont cependant leur envers, qui est une certaine monotonie de l'inspiration. et surtout du registre. Peu d'images jouant sur plusieurs claviers, projetant de multiples résonances. Le langage reste assez proche de la prose. C'est un langage à effets immédiats, sans fulgurations particulières. Et les poèmes se suivent sans surprise, articulant toujours le même constat de vie à refaire et de combats à subir.

À tout prendre, un premier recueil fort honnête, auquel manque peut-être un peu de fraîcheur.

Quand on est l'enfant d'une patrie inepte et suicidaire (le Québec), il est bon de trouver en soi, et dans l'amitié de quelques-uns, les ressources pour croire en l'excellence du monde et de cette vie. Jean-Marc Fréchette, auteur du Corps de l'infini (poèmes 1968-1985) ${ }^{5}$, nous propose l'évasion totale, la 
célébration d'une nature fraîche comme au premier jour, d'un Matin si pur / et dénué d'outils où Les âmes traversent le paysage. On imagine mal un ouvrier (cf. outils) dans semblable décor; mais les anges, les bergers et les moissonneurs s'y côtoient, tous gravitant autour de Meera, la Mère, l'Admirable, incarnation de cette Origine dont le poète poursuit inlassablement le rêve.

Je n'ai pas de penchant particulier pour la poésie mystique, qui substitue au réel d'aimables compensations. La poésie de Fréchette nous touche néanmoins par une indiscutable sincérité et de grands bonheurs d'expression. Très loin du lyrisme boursouflé d'un autre Fréchette, fameux en son temps. le chant de l'exultation est sobre, peu entaché de rhétorique. La vivacité des images est le principal atout du poème:

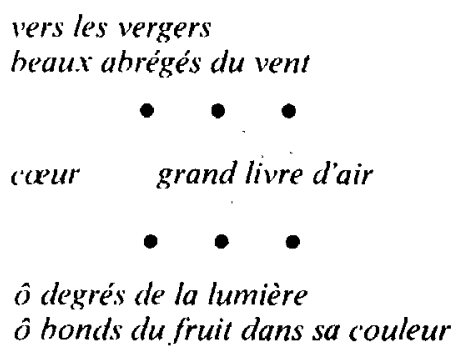

Certes, les grands mots simples ne sauraient donner naissance à des métaphores fracassantes, dont on comprendrait mal, du reste, la pertinence; mais il suffit que Fréchette nous redonne ces mots en leur pleine fraîcheur, réinvente l'«infini» avec l'enthousiasme et la probité d'un initié. pour qui le langage reçoit son sens et sa fonction d'une Transcendance. L'intention poétique souffre peut-être de cette surmotivation, mais elle y gagne aussi. Il reste, en chacun de nous, des nostalgies de merveilleux catholique que peut éveiller sans dommage l'évocation d'anges aux pieds de sel frais.

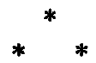

Alors que Fernande Saint-Martin écrivait "la fiction du réel ", attitude fort compatible avec un constructivisme littéraire, Claude Beausoleil nous propose "le réel de la fiction" dans son dernier livre aux dimensions, encore une fois, considérables: Il y a des nuits que nous habitons tous ${ }^{6}$.

Le réel de la fiction, cela veut dire que l'écriture s'invente à même le réel; que la nuit par exemple, tout comme la ville qui fut le thème de vastes poèmes de Beausoleil, est le lieu même, voire le sujet du texte, qu'elle est contemporaine du je dans son profus surgissement langagier. C'est tout un, pour Beausoleil, de dire la question de la nuit, l'écriture du livre et le vivre intime, de dire l'événement intérieur et "les effets du réel». De même que Hugo, dans ses grands poèmes de l'exil, rend visibles, à coups de formules inouies, les prodigieux linéaments du monde, de l'abî̀me, de l'ínconnu, Beau- 
soleil donne consistance à ce qu'on n'a jamais représenté, à cette histoire marquée que l'on ne raconte jamais. Il édifie un livre prismatique... autour du quotidien infini un livre pour comprendre le sens du noir, et certes, l'intention n'a ni la précision ni l'ampleur du projet hugolien, mais on $y$ trouve cet engagement entier qui caractérise aussi, dans le champ du roman. l'entreprise d'un Victor-Lévy Beaulieu.

Ce vaste recueil de deux cents pages est très construit à certains égards, avec sa structure répétitive - huit blocs comprenant chacun six divisions semblables - et très "mou" par d'autres côtés puisque l'acte d'écrire, d'une certaine façon, précède l'élaboration thématique (comme l'existence précède l'essence, dans l'ontologie de Sartre). 11 y a une bonne part de remplissage. Beausoleil ne pratique guère la peinture de chevalet: il lui faut des murs complets, et l'échelle où il travaille ne convient guère aux lectures myopes. attentives au détail. Très peu de bonheurs d'expression chez lui, plutôt des bonheurs de composition, de formulation au sens le plus large: j'écris dans une immense volupté ébloui des reprises et des formulations. Le lecteur peut et doit se laisser prendre par ce grand soulèvement de langage, habiter cette patrie des mots que le poète crée à son intention, avec la générosité, la chaleur, l'intelligence des formes du concret qui le caractérisent.

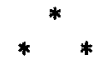

Un vers d'Eluard, Mon amour ton amour ton amour ton amour, ouvre et ferme le dernier recueil de Jean Charlebois, Tâche de naissance ${ }^{7}$. Dédaigneux, cette fois, des architectures complexes, le poète rassemble de courts poèmes en vers libres. Quatre sections les accueillent: "Le grand grand manque d'air", "Ne me touchez pas", "Truchement" et "L'immense" qui occupe à lui seul les trois cinquièmes du livre.

Charlebois est avant tout le chantre de l'amour, l'amour qu'on fait, et on ne lit pas sans malaise les poèmes pleins de bonne volonté qu'il a écrits contre la peine de mort. Déjà le titre ( Ne me touchez pas»), lointainement dérivé d'une parole de Jésus à Madeleine là pécheresse, est aux antipodes d'une thématique où les corps sont ouverts, voués les uns aux autres, et qui est la vraie veine du poète. Rien d'étonnant, d'ailleurs, si le mouvement du corps vers l'autre se matérialise fréquemment dans les yeux, le regard - qui est le véhicule constant du désir. Bien que l'érotique de Charlebois valorise de nombreux motifs, joue, langue, doigts, mains..., elle privilégie les yeux parce qu'ils sont capables de résumer tout le présent et de l'offrir en partage:

tes yeux sont des nappes blanches

où tu poses les fruits les douceurs et l'eau fraîche

où tu t'assembles autour de moi à contre-jour

ton regard d'enfant sans ombre et solitaire

outre mesure dans les allées de l'aeil

coule à fond dans les joncs de mes mains trop cernées

il est linnocence des herbes où les oiseaux vont mourir 
Deux isotopies se succèdent, l'isotopie domestique (nappes, nourritures) et l'isotopie naturelle (eau, herbes), donnant au poème sa continuité mais aussi son imprévu. Charlebois, tout en traitant des sujets șimples, sait déjouer les attentes et ouvrir des horizons soudains et immenses, par la seule grâce dụ désir souverain qui, dans le corps, vise et atteint la chair mais aussi la «belle âme".

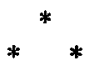

Un livre beau mais curieux, Instant-phénix, réunit des textes peu accessibles de Gatien Lapointe ${ }^{8}$. On a parlé d'édition pirate ${ }^{9}$, et le fait est que l'on n’y trouve ni copyright, ni mentions d'éditeur ou d'imprimeur, ni date de parution. Cette publication serait destinée à faire pression sur la famille et les amis du poète mort il y a deux ans, pour accélérer l'édition de son cuvre intégrale. Sur la couverture bleu nuit, on a reproduit quelques vers qui terminent un poème de 1956:

je vous redonnerai

en deux ou trois mots pirates

le denier du rêve

lor dur de la mort.

Un autre vers du même poème constitue aussi un bon commentaire de ce livre clandestin:

Nous ferons le projet d'une fraude extraordinaire.

Il y a de fait un côté fraudeur, pirate chez Gatien Lapointe, qui chante et vit le pays à partir de ses gestes premiers, impulsions anarchiques du coureur des bois accordées aux rougeoiements de braise du soleil sur le fleuve, à l'émiettement du sang dans les clairières de l'automne, au surgissement des mots premiers dans «le printemps du Québec». Le chantre du territoire est avant tout le nomade, l'homme du sang libre et du cri fondateur - fondateur dans la joyeuse dispersion - , le «clandestin de l'extraordinaire». Or, celui qui a si bien incarné, lors de la Révolution tranquille, l'humanisme nationaliste ouvert à l'universel (souvenons-nous que l'Ode au Saint-Laurent est précédée de J'appartiens à la Terre) n'a pas renié sa foi inspiratrice, quand la plupart des "poètes du pays" se sont recyclés du côté du formalisme ou de la contre-culture. En témoigne la "Chorégraphie d'un pays" (1980-1981), postérieure aux poèmes d'Arbre-radar ${ }^{10}$ où Lapointe explorait, avec l'enthousiasme à la fois brouillon et prudent qui. le caractérise, une anti-rhétorique très orientée vers la pure signifiance.

Instant-phénix a le mérite de faire apparaître à la fois la continuité des thèmes et des images, dans des textes qui vont de 1956 à 1981 , en même temps que la diversité des manières du poète. Et de nous rappeler la valeur et l'importance d'une œuvre qui compte parmi les réussites significatives de la poésie québécoise. 
La poésie de Josée Yvon, dans Filles-missiles ", est plus ramassée, plus incisive que dans ses précédents recueils et elle y gagne en force suggestive. On entrevoit une quotidienneté tragique, où le crime, la vénalité, l'abjection sont l'aura noire du vécu. Un arbitraire violent commande la composition du poème, qui se développe au hasard des connotations horribles, mais aussi parfois merveilleuses:

m'enracine sur le difficile
habite une gale
sorte de corset vert sale électrique
Peau d'Ane
n'importe où entre le ciment
pas de tendresse plus profonde
qu'un mal sournois
en perte de châle de réaction
dans l'oreiller des lumières bleues
qui réduit de mettre au monde
toute fille trop classique de rêve déchirée

Il y a d'abord ellipse du je (m'enracine , habite), réduction du langage à l'essentiel. Simultanément, le choix de la vie difficile est métaphorisé en enracinement, il y a intériorisation et conversion en vérité organique du destin mauvais. La demeure, où prend dimension le vécu, est une gale qui dit bien l'intériorisation de la spatialité et sa réduction à l'organique. tout en évoquant en filigrane le galetas (réduit misérable). Le corsage vert sale électrique allie le réalisme sordide et une dimension merveilleuse qui favorise le passage à Peau d'Âne - conte où la crasse fait justement bon ménage avec l'enchantement. Le reste du poème déploie plus explicitement le merveilleux (l'oreiller des lumières bleues) en contrepoint avec le tragique (fille de rêve... déchirée), l'isotopie vestimentaire (châle, oreiller, déchirée) prolongeant le corsage vert sale et donnant un tour concret au discours de l'exister (tendresse. mal sournois, réaction, mettre au monde).

L'obscurité du poème fait partie de sa violence, et les haillons d'images hantent le lecteur comme des remords.

C'est un titre particulièrement sombre que porte, sur sa couverture toute noire, le dernier recueil de Gilbert Langevin, Comme un lexique des abîmes ${ }^{12}$. Pourtant la tonalité d'ensemble du recueil n'est pas si désespérée, et la petite suite qui lui donne son nom commence par une profession d'énergie, en forme d'art poétique. La poésie, écrit Langevin, doit être Tangible. Vibrationnelle, éloignée aussi bien de la tradition (caducité) que de la nouveauté-pour-lanouveauté. Elle doit être poévie - on rejoint le vécrire de GalarneauGodbout. Le deuxième fragment chevauche joyeusement l'utopie: $\dot{A}$ quand la naissance d'une parousie remplie de racines communicantes? Le "lexique des abîmes" s'énonce abruptement dans le fragment suivant et introduit une thématique de l'encerclement et de la mort. 
Dans cette poésie du moi - un moi, certes, exemplaire puisque Langevin pratique, ou plutôt incarne la poésie comme un sacerdoce, non à la façon du poète maudit mais d'un famélique troubadour à la sagesse étrange - les mouvements d'espoir et de détresse se succèdent sans transition, peut-être parce que le discours du poème dit constamment l'essentiel, c'est-à-dire ce battement que font au plus profond de lêtre les pulsions de la vie et de la mort, l'une à l'autre enlacées. Bachelard n'écrivait-il pas que la contradiction est la loi de l'inconscient? Pour le poète dont le devenir a toujours du plomb dans l'être, homme blessé par une société sourde à ses appels, le destin s'énonce forcément en termes de noir et de blanc. Une certaine fantaisie, pourtant, fait parfois danser les significations, nous éloigne momentanément des sentiments extrêmes et prépare le renouvellement de leur expression. La vibration, à la fin, l'emporte et le noir est vidé de sa ténèbre, changé en sang. nouveau miracle de Cana:

sous les larmes rutile une encre sans tache

Pour terminer, je signale la réédition d'un classique de notre poésie, les Soirs rouges de Clément Marchand ${ }^{13}$, origine lointaine de la très actuelle poésie de la ville.

1. Fernande Saint-Martin. la Fiction du réel, poèmes 1953-1975. Montréal. l'Hexagone. 1985, $310 \mathrm{p}$.

2. François Charron. le Fait de vivre ou d'avoir yécu, Montréal, les Herbes rouges. 1986. $148 \mathrm{p}$.

3. Denise Desautels. la Répétition. Montréal, NBJ, 1986,60 p. L'Écran a été publié en 1983 (Saint-Lambert. le Noroît. 90 p.).

4. Christiane Frenette. Indigo nuit, Montréal, Leméac. 1986, 60 p.

5. Jean-Marc Fréchette. le Corps de l'infini. Montréal. Triptyque, 1986. 134 p.

6. Claúde Beausoleil. Il y a des nuits que nous habitons tous. Saint-Lambert et Pantin (France), le Noroît / le Castor astral, 1986, 196 p.

7. Jean Charlebois. Tâche de naissance, Saint-Lambert, Le Noroìt. 1986, 154 p.

8. Gatien Lapointe, Instant-phénix, sans nom d'éditeur, sans date, 84 p.

9. Jean Royer, «Une édition pirate de la poésie de Gatien Lapointe», le Devoir, le 14 juin 1986, p. C-3. Notons que les éditions de l'Hexagone annonçaient déjà, il y a six ans, un recueil de Gatien Lapointe sous le même titre.

10. Gatien Lapointe. Arbre-radar. Montréal. l'Hexagone. 1980, $150 \mathrm{p}$.

11. Josée Yvon. Filles-missiles. Trois-Rivières. Ėcrits des Forges. 1986. 72 p.

12. Gilbert Langevin. Comme un lexique des abimes. Trois-Rivières. Écrits des forges, 1986, 70 p.

13. Clément Marchand, les Soirs rouges. Montréal, Stanké, «10/10», 1986. 216 p. 